\title{
CLOSED MAPS AND THE CHARACTER OF SPACES
}

\author{
YOSHIO TANAKA
}

ABSTRACT. We give some necessary and sufficient conditions for the character of spaces to be preserved under closed maps.

Introduction. Recall the following result due to K. Morita and S. Hanai [11] or A. H. Stone [14]: Let $Y$ be the image of a metric space $X$ under a closed map $f$. Then $Y$ is first countable (or metrizable) if and only if every boundary $\partial f^{-1}(y)$ of the point-inverse $f^{-1}(y)$ is compact.

E. Michael [8] showed that every $\partial f^{-1}(y)$ is compact if $X$ is paracompact, and $Y$ is locally compact or first countable. For the Lindelöfness of $\partial f^{-1}(y)$ with $X$ metric, see [15].

For a space $X$ and $x \in X$, let $\chi(x, X)$ be the smallest cardinal number of the form $|\Re(x)|$, where $\Re(x)$ is a nbd base at $x$ in $X$. The character $\chi(X)$ of $X$ is defined as the supremum of all numbers $\chi(x, X)$ for $x \in X$. Let $f$ be a closed map from $X$ onto $Y$. First, we show that the character of $Y$ has an influence on the boundaries $\partial f^{-1}(y)$; indeed, they become Lindelöf or $\alpha$-compact by the situation of $Y$. Second, in terms of these boundaries, we give some necessary and sufficient conditions for the character of $X$ to be preserved under $f$.

We assume all spaces are regular and all maps are continuous and onto.

1. $\alpha$-compactness of the boundaries. We recall some definitions. A space $X$ is strongly collectionwise Hausdorff if, whenever $D=\left\{x_{\alpha} ; \alpha \in A\right\}$ is a discrete closed subset of $X$, there is a discrete collection $\left\{U_{\alpha} ; \alpha \in A\right\}$ of open subsets with $U_{\alpha} \cap D$ $=\left\{x_{\alpha}\right\}$. Every paracompact space is strongly collectionwise Hausdorff.

Let $\alpha \geqslant \omega_{0}$ and $\alpha^{+}$be the least cardinal number greater than $\alpha$. A space $X$ is $\alpha$-compact if every subset of $X$ of cardinality $\alpha$ has an accumulation point in $X$. A space $X$ is $\alpha$-Lindelöf if every open cover of $X$ has a subcover of cardinality $\leqslant \alpha$. Every $\alpha$-Lindelöf space is $\alpha^{+}$-compact.

A space $X$ is sequential if $F \subset X$ is closed in $X$ whenever $F \cap C$ is closed in $C$ for each compact metric subset $C$ of $X$. If we replace "compact metric subset" by "countable subset", then such a space is said to have countable tightness. Every sequential space is precisely the quotient image of a metric space [3].

Received by the editors March 23, 1983 and, in revised form, July 11, 1983.

1980 Mathematics Subject Classification. Primary 54A25, 54C10; Secondary 54B15, 54D20, 54D55.

Key words and phrases. Closed map, strongly collectionwise Hausdorff, $\alpha$-compact, $\alpha$-Lindelöf, sequential space, character. 
Following the terminology "c-sequential spaces" defined in [13], let us call $x \in X$ a c-sequential point in $X$ if, whenever $x$ is not isolated in a closed subset $F$ of $X$, there exists a sequence in $F-\{x\}$ converging to the point $x$. Every point of a sequential space is $c$-sequential.

Proposition 1.1. Let $f: X \rightarrow Y$ be a closed map with $X$ strongly collectionwise Hausdorff. If $y \in Y$ satisfies (1) or (2) below, then $\partial f^{-1}(y)$ is $\alpha$-compact.

(1) The point $y$ has a nbd system $\left\{V_{\beta} ; \beta<\alpha\right\}$ such that if $y_{\beta} \in V_{\beta}$ and the $y_{\beta}$ are all distinct, then $\left\{y_{\beta} ; \beta<\alpha\right\}$ has an accumulation point in $Y$.

(2) The point $y$ is c-sequential and $\chi(y, Y)<2^{\alpha}$.

Proof. Suppose $\partial f^{-1}(y)$ is not $\alpha$-compact. Then there exists a discrete collection $\left\{U_{\beta} ; \beta<\alpha\right\}$ of open subsets of $X$ meeting $\partial f^{-1}(y)$.

Case (1). There exists $x_{0} \in U_{0} \cap\left(f^{-1}\left(V_{0}\right)-f^{-1}(y)\right)$; hence $x_{0} \in U_{0}$ and $f\left(x_{0}\right) \in$ $V_{0}-\{y\}$. For $\beta<\alpha$, assume there exists a subset $F_{\beta}=\left\{f\left(x_{\gamma}\right) ; \gamma<\beta\right\}$ of $Y$ such that $x_{\gamma} \in U_{\gamma}, f\left(x_{\gamma}\right) \in V_{\gamma}-\{y\}$, and the $f\left(x_{\gamma}\right)$ are all distinct. Then, since $F_{\beta}$ is closed in $Y$ with $F_{\beta} \boxplus y$, there exists a nbd $V$ of $y$ with $V \cap F_{\beta}=\varnothing$. Thus there exists $x_{\beta} \in U_{\beta} \cap\left(f^{-1}\left(V_{\beta} \cap V\right)-f^{-1}(y)\right)$, hence $x_{\beta} \in U_{\beta}, f\left(x_{\beta}\right) \in V_{\beta}-\{y\}$, and $f\left(x_{\beta}\right) \notin F_{\beta}$. Then, by induction there exists a subset $F=\left\{f\left(x_{\beta}\right) ; \beta<\alpha\right\}$ of $Y$ such that $x_{\beta} \in U_{\beta}, f\left(x_{\beta}\right) \in V_{\beta}$ and the $f\left(x_{\beta}\right)$ are all distinct. Thus, $F$ is discrete in $Y$, but it has an accumulation point in $Y$. This is a contradiction. Hence $\partial f^{-1}(y)$ is $\alpha$-compact.

Case (2). Since the point $y$ is not isolated in $f\left(\overline{U_{\beta}}\right)$ for each $\beta<\alpha$, there exist sequences $C_{\beta}$ in $f\left(\overline{U_{\beta}}\right)-\{y\}$ converging to $y$. For $\gamma<\alpha$, assume there exists a pairwise disjoint collection $\left\{C_{\beta(\delta)} ; \delta<\gamma\right\}$ in $Y$ such that each $C_{\beta(\delta)}$ is some $C_{\beta}$ except at finitely many points. Since $\left\{C_{\beta(\delta)} ; \delta<\gamma\right\}$ is hereditarily closure-preserving, for each $\delta<\gamma,\left\{\beta ; C_{\beta} \cap C_{\beta(\delta)}\right.$ is infinite $\}$ is finite. Thus, there exists $\beta_{0}<\alpha$ such that $C_{\beta_{0}} \cap C_{\beta(\delta)}$ is finite for each $\delta<\gamma$. Then it follows that $C_{\beta_{0}} \cap \cup_{\delta<\gamma} C_{\beta(\delta)}$ is finite. Put $C_{\beta(\gamma)}=C_{\beta_{0}}-\cup_{\delta<\gamma} C_{\beta(\delta)}$. Then $C_{\beta(\gamma)} \cap C_{\beta(\delta)}=\varnothing$ for each $\delta<\gamma$. Hence, by induction there is a pairwise disjoint collection $\left\{C_{\beta(\gamma)} ; \gamma<\alpha\right\}$ in $Y$ such that each $C_{\beta(\gamma)}$ is assumed to be some $C_{\beta}$. Let $S=\cup_{\gamma<\alpha}\left(C_{\beta(\gamma)} \cup\{y\}\right)$. But, since $\left\{C_{\beta(\gamma)} \cup\right.$ $\{y\} ; \gamma<\alpha\}$ is a hereditarily-closure preserving closed cover of $S$, it is easy to show that $U \subset S$ is open (resp. closed) in $S$ whenever $U \cap\left(C_{\beta(\gamma)} \cup\{y\}\right)$ is open (resp. closed) in $C_{\beta(\gamma)} \cup\{y\}$ for each $\gamma<\alpha$. Then $\chi(y, S)=2^{\alpha}$; hence $\chi(y, Y) \geqslant 2^{\alpha}$. This is a contradiction. Hence, $\partial f^{-1}(y)$ is $\alpha$-compact.

COROllary 1.2. Let $f: X \rightarrow Y$ be a closed map with $X$ strongly collectionwise Hausdorff. Then $\partial f^{-1}(y)$ is a-compact if one of the following three properties is satisfied.

(1) $y$ has a nbd which is $\alpha$-compact.

(2) $\chi(y, Y) \leqslant \alpha$.

(3) $Y$ is sequential and $\chi(y, Y)<2^{\alpha}$.

Under Martin's Axiom (MA), using [7, Proposition 1.1] we have the following by Proposition 1.1 (Case (2)). 
Corollary 1.3 (MA). Let $f: X \rightarrow Y$ be a closed map with $X$ normal. If $Y$ has countable tightness, then $\partial f^{-1}(y)$ is countably compact if $\chi(y, Y)<2^{\omega_{0}}$. When $Y$ is especially sequential, we can omit the assumption (MA).

In terms of certain quotient ranges, we give another sufficient condition for the boundaries to be $\alpha^{+}$-compact (indeed, $\alpha$-Lindelöf). First, we state definitions.

A space $X$ is inner-one $A[\mathbf{1 0}]$ if, whenever $\left(A_{i}\right)$ is a decreasing sequence of subsets with $x \in \overline{A_{i}-\{x\}}$ (denoted by $\left(A_{i}\right) \downarrow x$ ), then there exist $a_{i} \in A_{i}$ such that $\left\{a_{i} ; i \in\right.$ $\left.\omega_{0}\right\}$ is not closed in $X$. Every $q$-space [8], or more generally every countably bi-quasi- $k$-space [9], is inner-one $A$. Recall that a space $X$ is perfect if every closed subset of $X$ is a $G_{\delta}$-set.

Proposition 1.4. Let $f: X \rightarrow Z$ be a closed map with $X$ paracompact and $Z$ sequential. Let $g: Y \rightarrow Z$ be a quotient map. Then $\partial f^{-1}(z)$ is $\alpha$-Lindelöf if $\partial g^{-1}(z)$ is $\alpha$-Lindelöf, and (1) or (2) below holds.

(1) $\left(2^{\alpha}<2^{\alpha^{+}}\right) \cdot \chi(Y) \leqslant 2^{\alpha}$, and either $Y$ is sequential or perfect.

(2) $Y$ is inner-one $A$.

Proof. Suppose $\partial f^{-1}(z)$ is not $\alpha$-Lindelöf. Since $\partial f^{-1}(z)$ is paracompact, $\partial f^{-1}(z)$ is not $\alpha^{+}$-compact. Since $Z$ is sequential, by the proof of Proposition 1.1, $Z$ contains a closed copy $S$ of the space obtained from the disjoint union of convergent sequences $\left\{C_{\beta} ; \beta<\alpha^{+}\right\}$by identifying all the limit points to the point $z$. Let $T=g^{-1}(S)$ and $h=g \mid T$. Since $S$ is closed in $Z, h$ is a quotient map.

Case (1). The space $S$ is Fréchet; that is, if $s \in \bar{A}$ in $S$, then there exists a sequence in $A$ converging to the point $s$. Hence, by the proof of [3, Proposition 2.3], the quotient map $h$ onto $S$ is a pseudo-open map (stated in the first paragraph of the next section). The closed subset $T$ of $Y$ is a sequential or perfect space with $\chi(T) \leqslant 2^{\alpha}$, and a closed subset $\partial_{T} h^{-1}(z)$ of $\partial_{Y} g^{-1}(z)$ is $\alpha$-Lindelöf. Thus, under $\left(2^{\alpha}<2^{\alpha^{+}}\right)$we have $\chi(z, S)<2^{\alpha^{+}}$by Proposition 2.4 in the next section. This is a contradiction. Hence, $\partial f^{-1}(z)$ is $\alpha$-Lindelöf.

Case (2). Assume the convergent sequences $C_{\beta}$ are subsets of $S$ (hence, $C_{\gamma} \cap C_{\delta}=$ $\{z\}$ if $\gamma \neq \delta)$, and let $T_{\beta}=h^{-1}\left(C_{\beta}-\{z\}\right)$ for each $\beta<\alpha^{+}$. Since each $T_{\beta}$ is not closed in $T$, there exists a subset $T^{*}=\left\{t_{\beta} ; \beta<\alpha^{+}\right\}$of $T$ with $t_{\beta} \in \overline{T_{\beta}}-T_{\beta}$. Suppose $\left|T^{*}\right|<\alpha^{+}$. Then there exists $\left\{\beta_{i} ; i \in \omega_{0}\right\}$ such that the $t_{\beta_{i}}$ are all the same point. Let $A_{i}=\cup_{j \geqslant i} T_{\beta_{j}}$ for each $i$. Then $\left(A_{i}\right) \downarrow t_{\beta_{0}}$.

Since $T$ is inner-one $A$, there exist $a_{i} \in A_{i}$ such that $A=\left\{a_{i} ; i \in \omega_{0}\right\}$ is not closed in $T$. Let $H_{i}=h^{-1}\left(C_{\beta_{i}}\right) \cap A$ for each $i$. Then each $h\left(H_{i}\right)$ is a finite subset of $C_{\beta_{i}}$ with $h\left(H_{i}\right) \boxplus z$. Let $V=\cup_{i \in \omega_{0}}\left(C_{\beta_{i}}-h\left(H_{i}\right)\right)$. Then $h^{-1}(V) \cap A=\varnothing$ and $V$ is a nbd of the point $z$ in $S^{\prime}=\bigcup_{i \in \omega_{0}} C_{\beta_{i}} \subset S$. Then $A$ is closed in $h^{-1}\left(S^{\prime}\right)$, hence in $T$. This contradiction implies the set $T^{*}$ has cardinality $\alpha^{+}$. Since $\partial h^{-1}(z)$ is an $\alpha^{+}$-compact subset of $T$ which contains the set $T^{*}$, there exists a point $t^{\prime} \in T$ and a subset $T^{\prime}$ of $T^{*}$ accumulating to the point $t^{\prime}$. Since $T$ is inner-one $A$, there exists a point $t \in T$ and a subset $\left\{b_{i} ; i \in \omega_{0}\right\}$ of $T^{\prime}$ accumulating to the point $t$. Let $b_{i}=t_{\beta_{i}^{\prime}}$ and $A_{i}^{\prime}=\cup_{j \geqslant i} h^{-1}\left(C_{\beta_{j}^{\prime}}-\{z\}\right)$ for each $i$. Then $\left(A_{i}^{\prime}\right) \downarrow t$. However, we have a contradiction by the same way as in the case where $\left|T^{*}\right|<\alpha^{+}$. Thus, $\partial f^{-1}(z)$ is $\alpha$-Lindelöf. 
Since every quotient image of a sequential space is obviously sequential, we have

Corollary 1.5. $\left(2^{\alpha}<2^{\alpha^{+}}\right)$. Let $f: X \rightarrow Z$ be a closed map with $X$ paracompact, and let $g: Y \rightarrow Z$ be a quotient map. Then $\partial f^{-1}(z)$ is $\alpha$-Lindelöf if $\partial g^{-1}(z)$ is also, and $Y$ is a sequential space with $\chi(Y) \leqslant 2^{\alpha}$. When $Y$ is especially first countable, we can omit the assumption $\left(2^{\alpha}<2^{\alpha^{+}}\right)$. Furthermore, we can replace " $\alpha$-Lindelöf" by " $\alpha^{+}$compact" if we replace paracompactness by strongly collectionwise Hausdorfness.

COROllaRY 1.6. Let $f_{i}: X_{i} \rightarrow Y(i=1,2)$ be closed maps with $X_{i}$ paracompact first countable. Then $\partial f_{1}^{-1}(y)$ is Lindelöf and compact if and only if $\partial f_{2}^{-1}(y)$ is, respectively.

2. Preservation of the character. A map $f: X \rightarrow Y$ is pseudo-open [1] if for any $y \in Y$ and any nbd $U$ of $f^{-1}(y), y \in$ int $f(U)$; equivalently, $f$ is hereditarily quotient, that is, $f \mid f^{-1}(S)$ is quotient for each $S \subset Y$ by [1, Theorem 1]. Every closed map or every open map is pseudo-open.

Lemma 2.1. Let $f: X \rightarrow Y$ be a pseudo-open map with $\chi(X) \leqslant 2^{\alpha}$. Then $\chi(y, Y) \leqslant 2^{\alpha}$ if $\partial f^{-1}(y)$ is an $\alpha$-Lindelöf space of cardinality $\leqslant 2^{\alpha}$.

Proof. Since $\chi(X) \leqslant 2^{\alpha}$ and $\left|\partial f^{-1}(y)\right| \leqslant 2^{\alpha}$, there is an open collection of of cardinality $\leqslant 2^{\alpha}$ such that for $x \in \partial f^{-1}(y)$ and a nbd $V$ of $x$ in $X, x \in B \subset V$ for some $B \in \mathscr{B}$. Let $U$ be a nbd of $y$ in $Y$. Since $\partial f^{-1}(y) \subset f^{-1}(U)$ and $\partial f^{-1}(y)$ is $\alpha$-Lindelöf, there is a subfamily $\mathscr{B}^{\prime}$ of $\mathscr{B}$ with $\left|\mathscr{B}^{\prime}\right| \leqslant \alpha$ such that $\partial f^{-1}(y) \subset \cup \mathscr{B}^{\prime} \subset$ $f^{-1}(U)$. Thus, $y \in$ int $f\left(\cup \mathfrak{B}^{\prime}\right) \cup f\left(\right.$ int $\left.f^{-1}(y)\right) \subset U$. Hence, $\chi(y, Y) \leqslant 2^{\alpha}$.

EXAMPLE 2.2. In Lemma 2.1, that $f$ is pseudo-open is essential. Indeed, let $X_{1}=D \cup\{\infty\}$ be the one-point compactification of a discrete space $D$ of cardinality $\alpha$. For each $d \in D$, let $I_{d}=[0,1] \times\{d\}$ be a copy of the closed unit interval $[0,1]$, and let $X_{2}$ be the disjoint union of $\left\{I_{d} ; d \in D\right\}$. Then the space $Y$ obtained from the disjoint union $X$ of $X_{1}$ and $X_{2}$ by identifying $d \in X_{1}$ to $(1, d) \in X_{2}$ for each $d \in D$ is the quotient finite-to-one image of a paracompact space $X$ with $\chi(X)=\alpha$, but $\chi(\infty, Y)=2^{\alpha}$.

The following lemma is due to Juhász [5] (cf. [4]) for Case (1), and Arhangel'skiı̆ [2] for Case (2).

LemMa 2.3. Let $X$ satisfy (1) or (2) below. Then $|X| \leqslant 2^{\alpha}$.

(1) Hereditarily $\alpha$-Lindelöf space.

(2) Sequential $\alpha$-Lindelöf space of character $\leqslant 2^{\alpha}$.

Every $\alpha$-Lindelöf perfect space is obviously hereditarily $\alpha$-Lindelöf. Thus, by Lemmas 2.1 and 2.3, we have

Proposition 2.4. Let $f: X \rightarrow Y$ be a pseudo-open map with $\chi(X) \leqslant 2^{\alpha}$. Then $\chi(y, Y) \leqslant 2^{\alpha}$ if $\partial f^{-1}(y)$ is $\alpha$-Lindelöf, and either $X\left(\right.$ or $\left.\partial f^{-1}(y)\right)$ is sequential or perfect.

The following example ${ }^{1}$ shows that the sequentiality or perfectness of $X$ (or $\left.\partial f^{-1}(y)\right)$ in the previous proposition is essential even if $f$ is perfect.

\footnotetext{
'This was suggested by $G$. Gruenhage.
} 
EXAMPLE 2.5. For each $\alpha \geqslant \omega_{0}$, there is a perfect map $f: X \rightarrow Y$ such that $X$ is a paracompact space with $\chi(X)=\alpha$ and $Y$ is a Fréchet space with $\chi(Y)=2^{\alpha}$. Indeed, let $K_{i}=K \times\{i\}(i=0,1)$ be copies of a compact space $K$ with $|K|=2^{\alpha}$ and $\chi(K)=\alpha$ (e.g., let $K=I^{\alpha}$, where $I$ is the closed unit interval). Let $X=K_{0} \cup K_{\text {I }}$ be the Alexandorff Double of $K$; that is, each point of $K_{1}$ is isolated, and $V \times\{0,1\}-\{(x, 1)\}$ is a nbd of $(x, 0)$ in $K_{0}$, where $V$ is a nbd of $x$ in $K$. Then the space $Y$, obtained from $X$ by identifying $K_{0}$ to a single point, is the closed image of a compact space $X$ with $\chi(X)=\alpha$. But $Y$ is the one-point compactification of a discrete space of cardinality $2^{\alpha}$. Thus, $Y$ is a Fréchet space with $\chi(Y)=2^{\alpha}$.

REMARK 2.6. Not every perfect image of a first countable space $X$ is first countable even if $X$ is a compact space (in the above example, put $K=I$ ), or a $\sigma$-space (e.g., see [6, Example 4.3]). Thus, in Proposition 2.4, we cannot replace " $2^{\alpha}$ " by " $\omega_{0}$ ", even if $f$ is a perfect map.

Every closed image of a sequential space is sequential, and every $\alpha^{+}$-compact paracompact space is $\alpha$-Lindelöf. Thus, combining Proposition 2.4 with Corollary 1.2 (Case (3)), we have

THEOREM 2.7. $\left(2^{\alpha}<2^{\alpha^{+}}\right)$. Let $f: X \rightarrow Y$ be a closed map with $X$ a paracompact sequential space with $\chi(X) \leqslant 2^{\alpha}$. Then every $\partial f^{-1}(y)$ is $\alpha$-Lindelöf if and only if $\chi(Y) \leqslant 2^{\alpha}$.

Corollary 2.8. $\left(2^{\alpha}<2^{\alpha^{+}}\right)$. Let $f: X \rightarrow Y$ be a closed map with $X$ a paracompact first countable space. Then every $\partial f^{-1}(y)$ is $\alpha$-Lindelöf if and only if $\chi(Y) \leqslant 2^{\alpha}$.

As a generalization of $\sigma$-spaces and paracompact $M$-spaces, K. Nagami [12] defined strong $\Sigma$-spaces (he also defined $\Sigma$-spaces). For the definition of strong $\Sigma$-spaces, see Definition 1.1 (or Lemma 1.4) in [12].

Lemma 2.9. Let $\alpha$ be a cardinal number with $\operatorname{cf}(\alpha)>\omega_{0}$. Then every $\alpha$-compact strong $\Sigma$-space is $\beta$-Lindelöf for some $\beta<\alpha$.

Proof. Let $X$ be a strong $\Sigma$-space. Then there exists a cover $\mathcal{K}$ of compact subsets of $X$ and a $\Sigma$-net $\mathscr{F}=\cup_{i \in \omega_{0}} \mathscr{F}_{i}$ for $X$ such that each $\mathscr{F}_{i}$ is a locally finite closed cover of $X$, and if $K \subset U$ with $K \in \mathscr{K}$ and $U$ open, then $K \subset F, \subset U$ for some $F \in \mathscr{F}$. If $X$ is $\alpha$-compact, by $\operatorname{cf}(\alpha)>\omega_{0}$, the $\Sigma$-net $\mathscr{F}$ has cardinality $\leqslant \beta$ for some $\beta<\alpha$. Then it is easy to show that $X$ is $\beta$-Lindelöf.

THEOREM 2.10 (MA). Let $f: X \rightarrow Y$ be a closed map with $X$ a paracompact $\Sigma$-space with $\chi(X) \leqslant 2^{\omega_{0}}$. Suppose $X$ is sequential or perfect. Then every $\partial f^{-1}(y)$ is $2^{\omega_{0}}$-compact if and only if $\chi(Y) \leqslant 2^{\omega_{0}}$.

Proof. The "if" part follows from Corollary 1.2 (Case (2)).

"Only if": Since every $\partial f^{-1}(y)$ is a strong $\Sigma$-space, by Lemma $2.9, \partial f^{-1}(y)$ is $\beta$-Lindelöf for some $\beta<2^{\omega_{0}}$. We remark that every $\beta$-Lindelöf perfect space is hereditarily $\beta$-Lindelöf. Thus, since $\chi(X) \leqslant 2^{\omega_{0}}=2^{\beta}, \chi(Y) \leqslant 2^{\omega_{0}}$ by Proposition 2.4 . 
COROllary 2.11 (MA). Let $f: X \rightarrow Y$ be a closed map with $X$ a paracompact space with $\chi(X) \leqslant 2^{\omega_{0}}$. Suppose $X$ is a $\sigma$-space or a sequential $M$-space. Then every $\partial f^{-1}(y)$ is $2^{\omega_{0}}$-compact if and only if $\chi(Y) \leqslant 2^{\omega_{0}}$.

\section{REFERENCES}

1. A. V. Arhangel'skiī, Some types of factor mappings, and the relations between classes of topological spaces, Soviet Math. Dokl. 4 (1963), 1726-1729.

2. On the cardinality of bicompacta satisfying the first axiom of countability, Soviet Math. Dokl. 10 (1969), $951-955$.

3. S. P. Franklin, Spaces in which sequences suffice, Fund. Math. 57 (1965), 107-115.

4. J. de Groot, Discrete subspaces of Hausdorff spaces, Bull. Acad. Polon. Sci. Sér, Sci. Math. Astronom. 13 (1965), 537-544.

5. I. Juhász, Cardinal functions in topology, Math. Centrum, Amsterdam, 1971.

6. D. J. Lutzer, Semimetrizable and stratifiable spaces, Topology Appl. 1 (1971), 43-48.

7. V. I. Malyhin and B. E. Šapirovskĩ,, Martin's axiom and properties of topological spaces, Soviet Math. Dokl. 14 (1973), 1746-1751.

8. E. Michael, $A$ note on closed maps and compact sets, Israel J. Math. 2 (1964), 173-176.

9. __ A quintuple quotient quest, Topology Appl. 2 (1972), 91-138.

10. E. Michael, R. C. Olson and F. Siwiec, A-spaces and countably bi-quotient maps, Dissertationes Math. (Rozprawy Mat.) 133 (1976), 4-43.

11. K. Morita and S. Hanai, Closed mappings and metric spaces, Proc. Japan Acad. 32 (1956), 10-14.

12. K. Nagami, $\Sigma$-spaces, Fund. Math. 65 (1969), 169-192.

13. D. V. Rančin, Tightness, sequentiality and closed coverings, Soviet Math. Dokl. 18 (1977), 196-200.

14. A. H. Stone, Metrizability of decomposition spaces, Proc. Amer. Math. Soc. 7 (1956), 690-700.

15. Y. Tanaka, Closed maps on metric spaces, Topology Appl. 11 (1980), 87-92.

Department of Mathematics, Tokyo Gakugei University, 4-1-1, Nukuikita-machi, Koganei-Shi, TOKYO, JAPAN 